\title{
TEXTURE EVOLUTION DURING THE DRAWING OF LOW CARBON STEEL
}

\author{
C. SCHUMAN ${ }^{1,2}$, C. ESLING ${ }^{1}$, M. J. PHILIPPE ${ }^{1}$, \\ M. HERGESHEIMER ${ }^{2}$, M. JALLON ${ }^{2}$ AND A. LEFORT ${ }^{2}$ \\ ${ }^{\prime} L M 2 P-I S G M P$, Ile du Saulcy, F-57045 METZ Cedex 01 \\ ${ }^{2}$ UNIMETAL Recherche, BP 140, F-57360 AMNEVILLE
}

(Received 15 November 1993; Received in final form 15 April 1994)

\begin{abstract}
This study deals with the texture evolution during drawing of interstitial-free low carbon steels under different conditions to study the possible influence of the drawing direction, deformation rate and metal/ die friction coefficient. The drawing has been carried out without intermediary annealing, with constant die angle and deformation rate per pass. In all cases, a $<110>$ fibre texture has been observed at the early stage of deformation (a few percents). The drawing direction, whether alternate or unidirectional, has little effect on texture. Slight differences only in the intensity of peaks on pole figures (PFs) are noted. Alternate drawing leads to higher drawing limits. The grain size affects both the texture and the mechanical properties, which are improved by fine grains. For industrial drawing, i.e. at a high deformation rate, no texture gradient has been clearly observed. Nevertheless, slight differences have been noted in the PF intensities, with generally a slightly sharper texture in the core, compared to the surface. The microhardness tests show no hardness gradient. In slow drawing (low deformation rate), there is a weak texture gradient which disappears at larger deformation. In order to visualize the influence of the metal/die friction, we used a material covered with copper. Results show that at a given reduction rate, the material covered with copper shows peak intensities on the (110) PF which are half these of a material drawn under conventional conditions. The drawing textures of BCC materials always present a $<110>$ fibre texture. A modeling of the texture evolution during drawing has also been carried out using the Taylor model.
\end{abstract}

KEY WORDS: Wire-drawing, drawing, IFS, low-carbon steel, texture, Taylor model.

\section{INTRODUCTION}

The study deals with the crystallographic texture evolution during the drawing of different interstitial-free low carbon steels (pure ferritic structure). Emphasis is put on texture variations and possible texture gradients about which the published results appear to be contradictory. The selection of the grades has been made to cover various mechanical and metallurgical characteristics. For the interstitial-free steels (IFS), the alloying of titanium allows to trap nitrogen (to avoid ageing after hardening), and to trap carbon (to avoid ageing after quenching - no carbon in oversaturation in the ferrite). In these alloys there is actually only one pure ferritic phase with titanium carbonitride precipitates. Different drawing conditions (drawing direction, deformation rate, metal/ die friction) and metallurgical conditions (two different grain sizes) have been studied to investigate a possible effect on the texture and the mechanical properties. In addition, mechanical tests have been carried out to correlate with the texture results. The Taylor 
model has been used in order to assess the gliding systems activated during deformation and to modelize the texture evolution of these materials during drawing.

\section{MATERIALS}

\section{II-1 Drawing process}

Drawings have been carried out with constant reduction rates (20\% per pass) and die angles $\left(12^{\circ}\right)$ using the test equipment of Unimetal (single-pass equipment) for materials A, B, C. Material A shows large grains $(30 \mu \mathrm{m})$, material B small grains $(10 \mu \mathrm{m})$. A and $B$ have both undergone an alternate drawing. Material $C$ which is the same as material $\mathrm{B}$ has undergone a unidirectional drawing. Material D which is the same as material $\mathrm{B}$ has undergone drawing at low speed. In this case the drawing process is slightly different. Materials A, B, and C are all three taken from the same cast. Material $\mathrm{E}$ is first covered with copper at high temperature, then drawn. As this drawing process is made on the industrial level, we could only obtain the material in two states, the 8. $\mathrm{mm}$ as-drawn machine wire and the $2 . \mathrm{mm}$ wire before annealing. For $\mathrm{A}, \mathrm{B}, \mathrm{C}$, $\mathrm{D}$, samples have been cut at each deformation stage. Drawing of $\mathrm{A}, \mathrm{B}, \mathrm{C}$ has been carried out up to the drawability limit without intermediary annealing. Materials A, $\mathrm{B}, \mathrm{C}$ underwent drawing starting with a diameter of $8 . \mathrm{mm}$ (as-drawn machine wire) and up to $0.6 \mathrm{~mm}$ for $\mathrm{A}$ (i.e. $99.44 \%$ reduction), $0.5 \mathrm{~mm}$ for B (i.e. $99.65 \%$ reduction) and $0.76 \mathrm{~mm}$ for $\mathrm{C}$ (i.e. $99.12 \%$ reduction).

\section{II-2 Pole Figure (PF) measurements}

Let us remind that Pole Figures (PFs) give the distribution of the normals to the crystallographic planes $(h k l)$ in the sample coordinate frame and can be directly measured by means of a four-circle diffractometer. The PF measurement is commonly performed on flat samples, but is more difficult for more complex shapes as it is the case for wires. Different methods can be used to measure PFs on wires:

1. by forming a close-packed single layer of wires and filling the interstices between them with an absorbing powder ( $\mathrm{Pb}$ type)

2. by embedding a close-packed single layer of wires in a resin and further polishing it up to a flat surface

3. by drilling a hole into the wire center and by further etching the hollow cylinder to a thin sheet to be unrolled elastically.

All these methods only apply to wires with large diameters. An alternative method which applies to thinner wires consists in setting these wires in a close-packed single layer. In this case, it will be necessary to correct the obtained intensities (geometry and absorption correction) to account for the difference in the diffracting behaviour with respect to a flat sample. These correction relationships (Langouche et al. (1989), Montesin et al. (1990)) of the diffracted intensities (effect of the wire geometry and absorption) restrict their use to wires with a diameter less than $3 . \mathrm{mm}$.

As the latter technique could only apply to the thinner wires, the measurements of all the PFs have been made systematically on the cross-sections of the wires studied in this work, the diameters of which range from $8 . \mathrm{mm}$ to $0.5 \mathrm{~mm}$. For wires with smaller diameters, composite samples have been prepared by assembling the wires into 


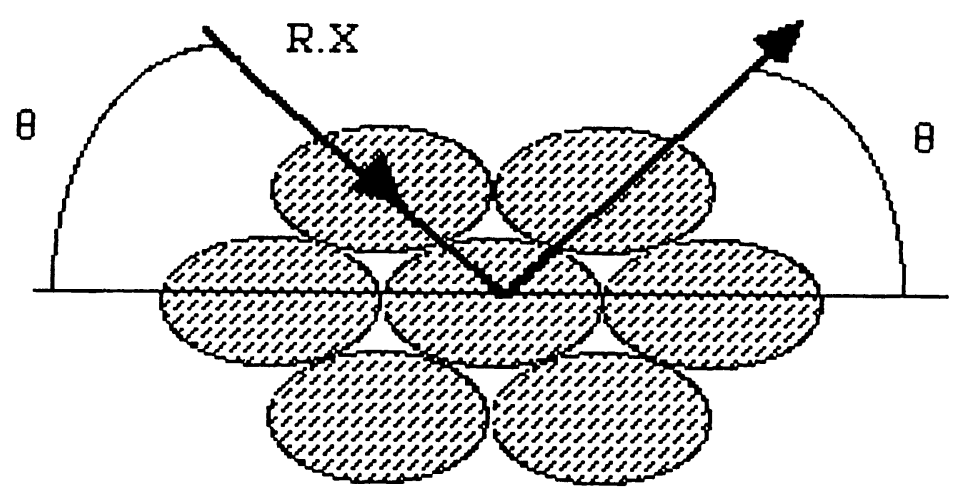

Figure 1 Bundle of wires.

bundles before cutting the cross section (Figure 1) to increase the diffracting volume. For each studied sample, three incomplete reflection pole figures (110), (200) and (112) have been measured on the wire cross-sections using the Schulz X-ray reflection technique.

When the PFs are measured on the cross-sections, the drawing axis, which is the rotational symmetry axis, is perpendicular to the projection plane of the pole figure. The pole figures measured in this setting show clearly the corresponding rotational fibre symmetry. However, in the literature PFs have often been measured on the wires surface. This corresponds to a different setting with the wire axis in the projection plane, parallel to the conventional "rolling direction" of the sample coordinate frame for rolled sheets. Three-dimensional texture analysis has been made using the series expansion method according to H. J. BUNGE (1969). The programmes of texture analysis were extended to include a possible transformation of the sample coordinate frame. Thus, although all the experimental PFs have been measured on the cross-sections, it was possible to obtain the ODF and the complete recalculated PFs in either one or the other setting of the sample coordinate frame, with the wire axis in the normal direction (as measured) or with the wire axis in the conventional "rolling direction" (Figures 2-5). This transformation is described by a simple rotation in the Euler space with the following Euler angles $(\pi / 2, \pi / 2, \pi / 2)$.

\section{RESULTS}

III-1 Effect of the grain size and of the drawing mode

\subsection{Effect of the grain size (Material A, B)}

The PFs obtained for material A and B are similar in shape but the pole densities of material A are lower than those of B. Indeed, the curves presenting the maximal (110) PF-intensities versus the deformation degree (Figure 6) show that the maximal (110) PF-intensities are increasing with the deformation degree, with systematically weaker values for material $A$ than for $B$, up to a reduction rate of 4 . For reduction rates higher than 4., the dispersions observed on Figure 6 can be attributed to the difficulty in setting wires with smaller diameters rigorously parallel when preparing samples from bundles of wires. 


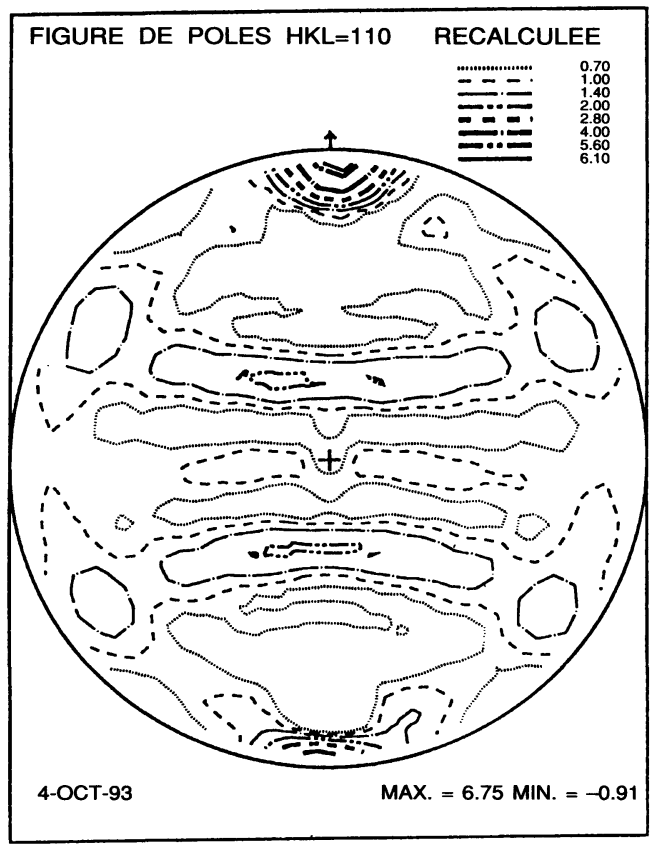

Figure 2 (110) recalculated PF of a thin wire surface without any correction.

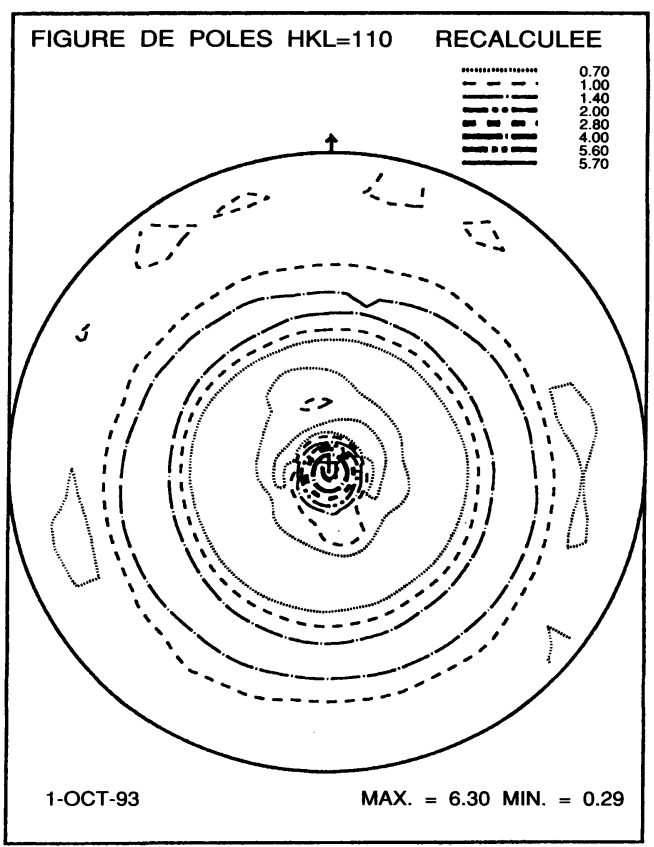

Figure 3 (110) recalculated PF of a thin wire section. 


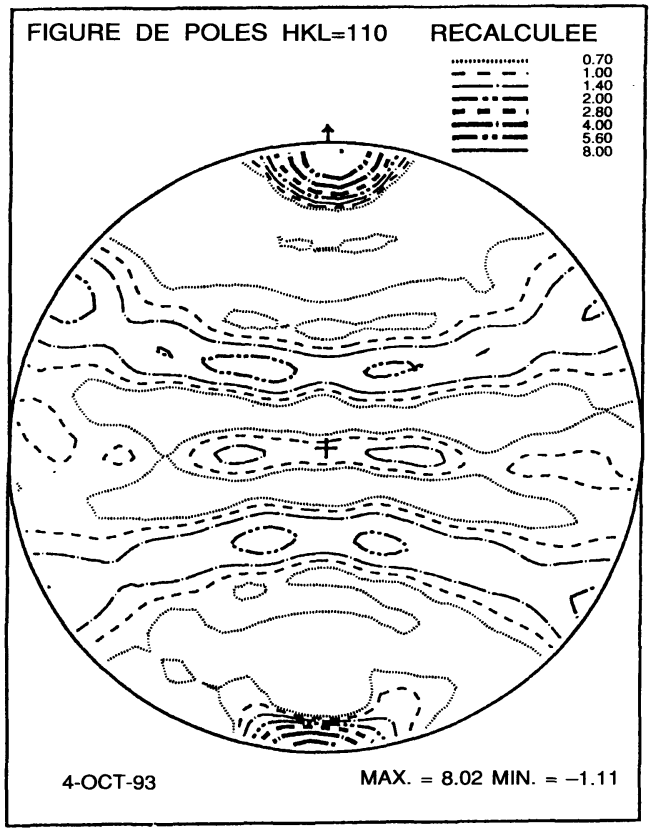

Figure 4 Same figure as Figure 2 with geometry correction.

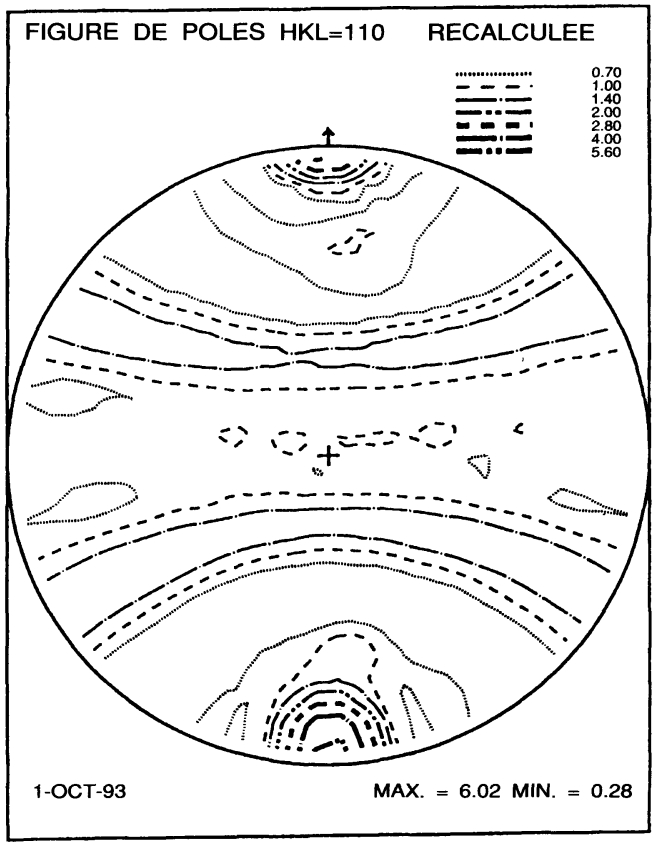

Figure 5 Same figure as Figure 3 after a transformation of the sample coordinate frame (Wire axis // to arrow). 


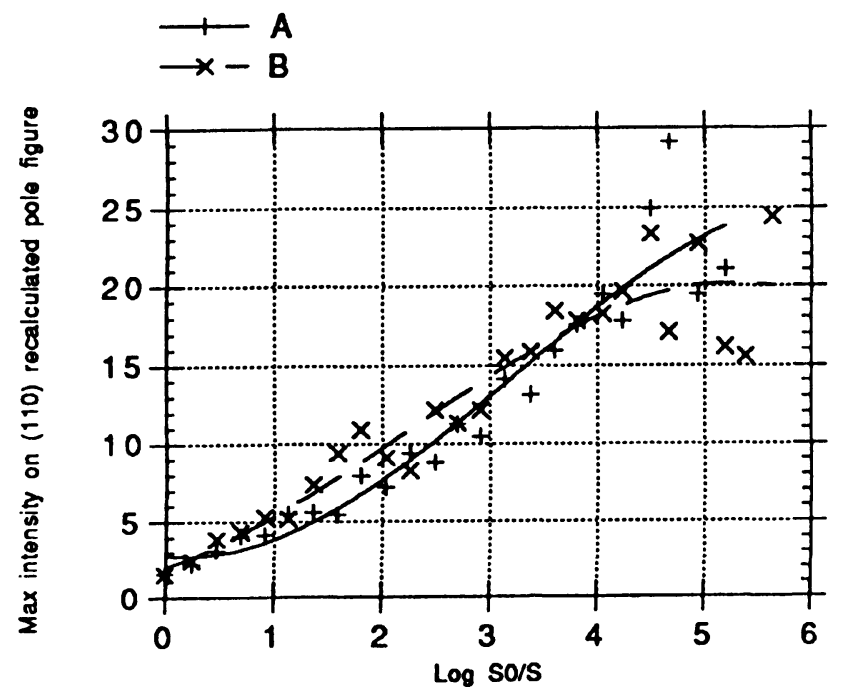

Figure 6 Max intensity on (110) recalculated PF versus $\varepsilon$ for A and B materials.

Concerning the mechanical properties, the differences observed on the curves representing the yield stress versus the deformation degree for materials A and B (Figure 7) can be directly linked to the difference in the initial grain size between both materials. Material B with the smaller grain size always presents a higher yield stress than material A with the larger grain size. This behaviour is in accordance with the Hall-Petch law (Embury et al. (1966), Lankford (1970)) which predicts that the yield stress varies according to the inverse square root of the grain size.

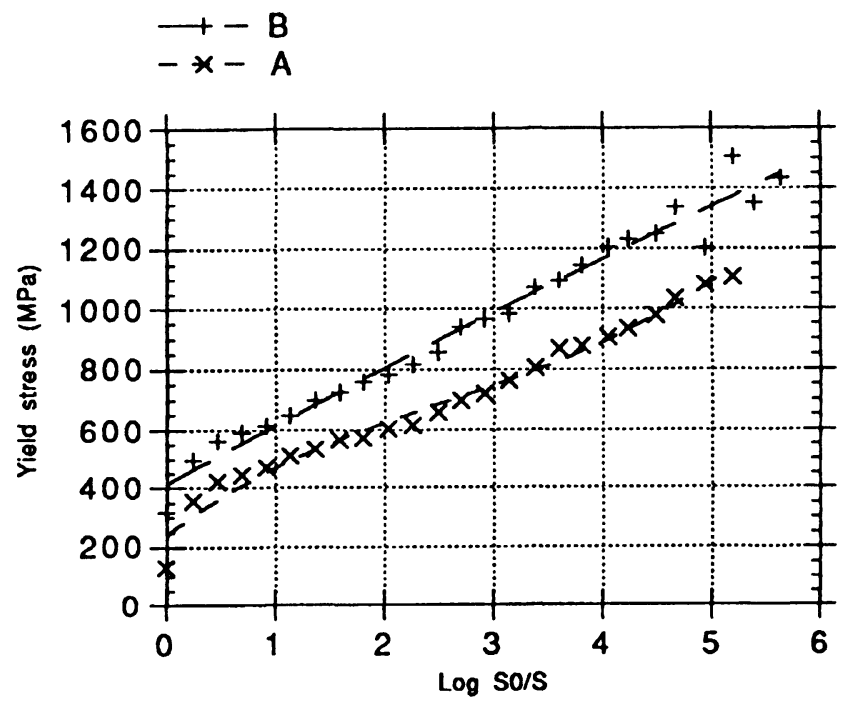

Figure 7 Yield stress versus $\varepsilon$ for A and B materials. 
1.2. Effect of drawing mode: alternate versus unidirectional (Material B, C)

Whether the drawing is alternate (Material B) or unidirectional (Material C) has no effect on the mechanical properties. Indeed, the curves representing the yield stress versus the deformation degree are exactly superimposed (see Figure 8) for materials $\mathrm{A}$ and $\mathrm{B}$.

The textures obtained are also quite similar as can be seen in Figure 9 presenting the curves of maximal (110) PF-intensities versus the deformation degree, on which only slight differences can be observed. However, for deformations higher than $90 \%$,

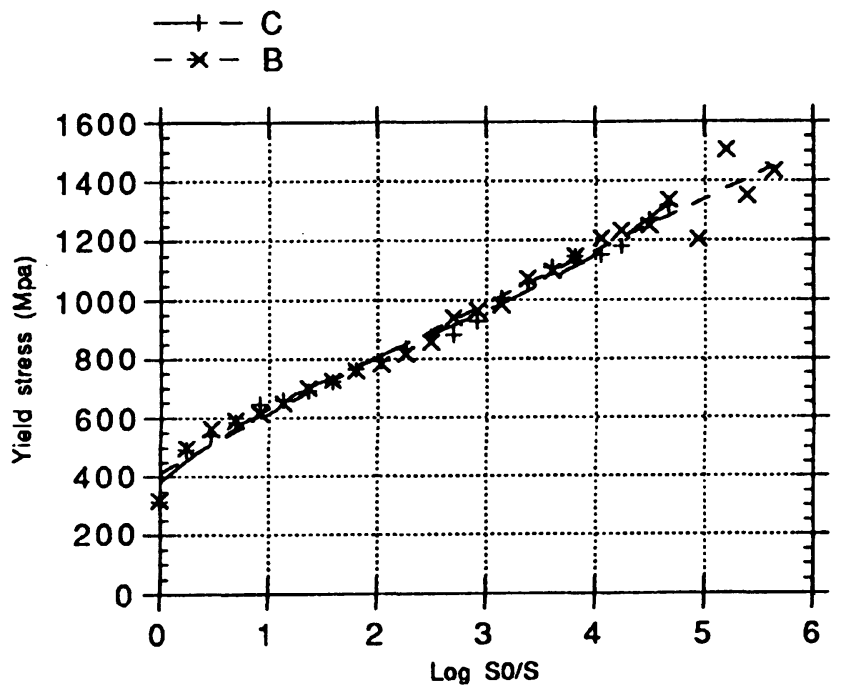

Figure 8 Yield stress versus $\varepsilon$ for $\mathrm{B}$ and $\mathrm{C}$ materials.

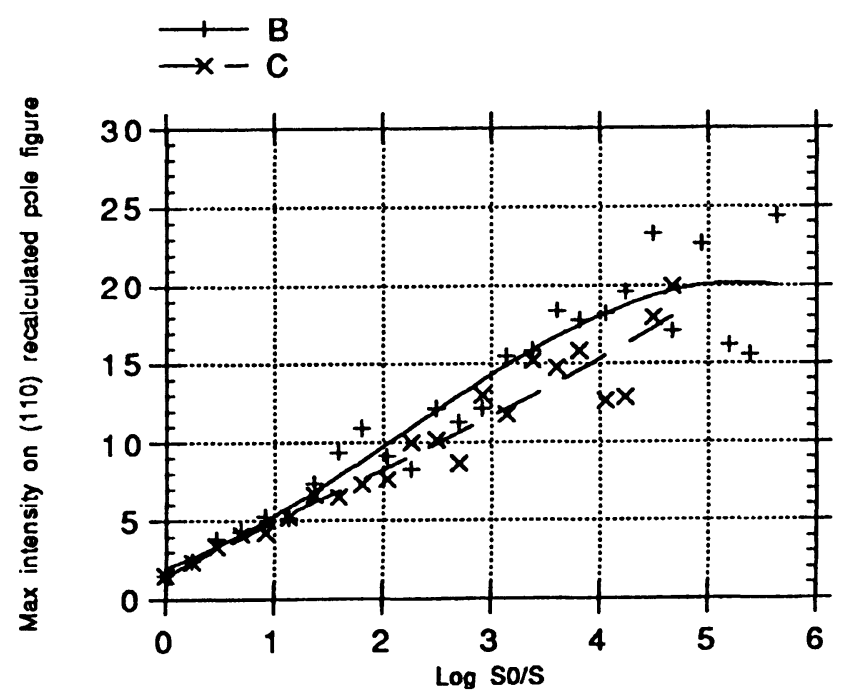

Figure 9 Max intensity on (110) recalculated PF versus $\varepsilon$ for B and C materials. 
the maximal pole densities observed on the recalculated (110) PFs are higher for B. In parallel, the dispersion in the represented points on which the interpolated curve is based increases. As previously, this dispersion at higher deformation degrees may be attributed to the increasing difficulty in the sample preparation.

The alternate drawing makes it possible to obtain a greater reduction in section before reaching the drawability limit. Indeed, the drawing limit is already reached at 0.76 diameter for material $\mathrm{C}$, whereas it is only reached at 0.5 diameter for material $\mathrm{B}$ with 4 more drawing passes than the material unidirectionally drawn.

\section{III-2 Effect of the drawing speed (Material C, D)}

Figure 10 represents the maximal PF density on the (110) recalculated PFs versus the deformation $\varepsilon$ for material $\mathrm{C}$ drawn at industrial speed and material $\mathrm{D}$ drawn at a low speed. For deformations smaller than 2., the two curves are superimposed so that there are no sizeable differences. A gap appears for deformations greater than 2 . The fact we did not find differences is probably due to the deformation rate per pass which is larger for D than for C. Thus the effects specifically due to the drawing speed could have been compensated by the different deformation rate per drawing pass. Montesin also obtained similar results (Montesin et al. (1991)) showing no effect of the strain rate on the texture (in the range studied).

\section{III-3 Effect of the die/metal friction (Material C, E)}

The copper-coated wire is obtained by hot corolling a steel wire covered with a thin $\mathrm{Cu}$ sheet. We have only two states, respectively the $8 . \mathrm{mm}$ machine wire and the 2. $\mathrm{mm}$ wire before annealing.

When comparing the recalculated (110) PF densities of the latter 2. mm covered wire E (Figure 11) with those of the $2.09 \mathrm{~mm}$ wire C (Figure 12), we note that the PF densities for $\mathrm{C}$ are about 1.5 times higher than for $\mathrm{E}$.

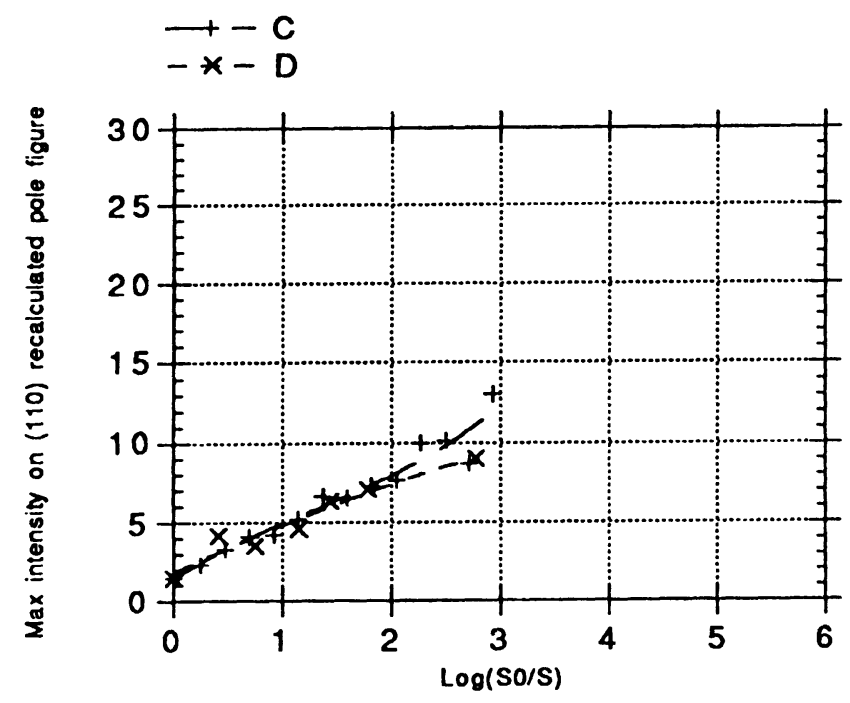

Figure 10 Max intensity on (110) recalculated PF versus $\varepsilon$ for $C$ and D materials. 


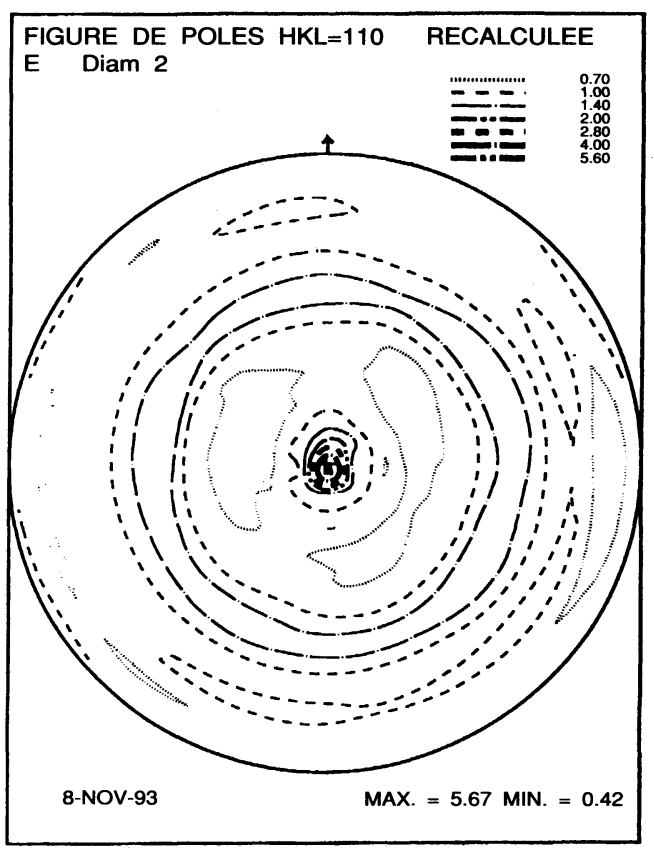

Figure 11 (110) recalculated PF for a $2 . \mathrm{mm}$ wire of material with copper coating.

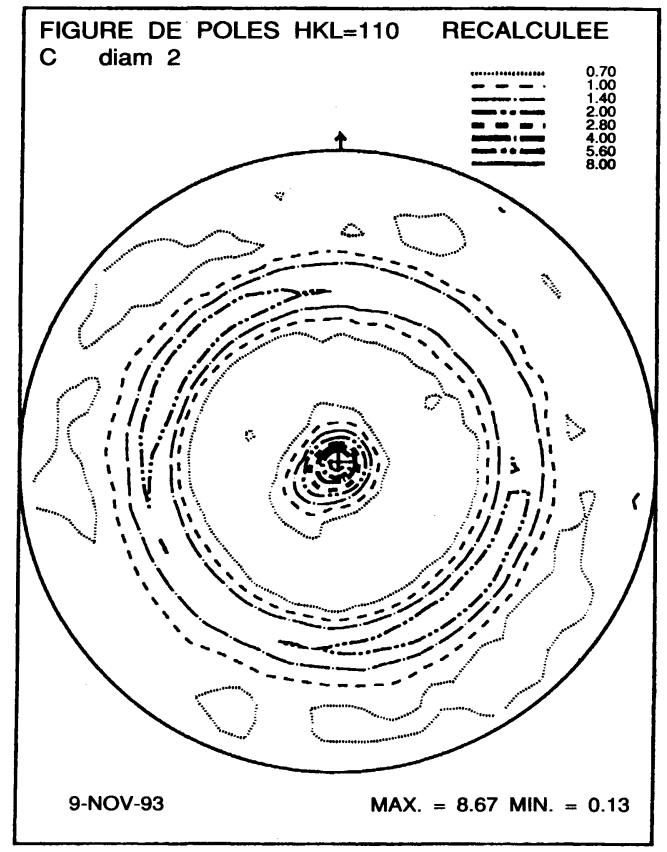

Figure 12 (110) recalculated PF for a $2 . \mathrm{mm}$ wire of $\mathrm{C}$ material. 


\section{III-4 Texture gradient (Materials A, B, C and D)}

Special attention has been paid to the study of a possible radial texture gradient in the wires from the surface to the core. The results reported in the literature seem to be contradictory on this point (Montesin et al. (1991)). For all the studied samples, the wires have been thinned down from their initial diameter in two steps, first by machining and then by chemical dissolution. The obtained results are shown in Figures 13 to 20 . Each figure simultaneously presents the maximal (110) PF density and the texture index versus the wire thinning ratio, i.e. the ratio of the current diameter $D$ to the initial one $D_{\text {ini }}$. In this scale, 1 corresponds to the external surface of the initial wire and 0 corresponds to the core of the wire (wire axis). The general impression given by these results is that the wires show no or small texture gradient, which is thus not easily identifiable. In parallel, the results of the measurements in microhardness present only a small hardness gradient along the wire radius, which is in accordance with the very small texture gradients we found.

As already mentioned, the drawing textures only vary to a small extent according to defined deformation conditions. It may be interesting to model the evolution of the texture of drawn wires in order to understand the mechanisms accomodating the plastic strain.

\section{III-5 Modelling of the texture evolution}

The texture evolution by drawing has been modelized with a Full-Constrained Taylor model. For the simulation, the starting texture was not the random one, but rather the actual experimental texture of materials $A$ and $B$ with $8 \mathrm{~mm}$ diameter. The texture has been determined by $\mathrm{X}$-ray PF inversion and the obtained Orientation Density Function (ODF) has been discretized by 948 individual orientations for A and 999 for

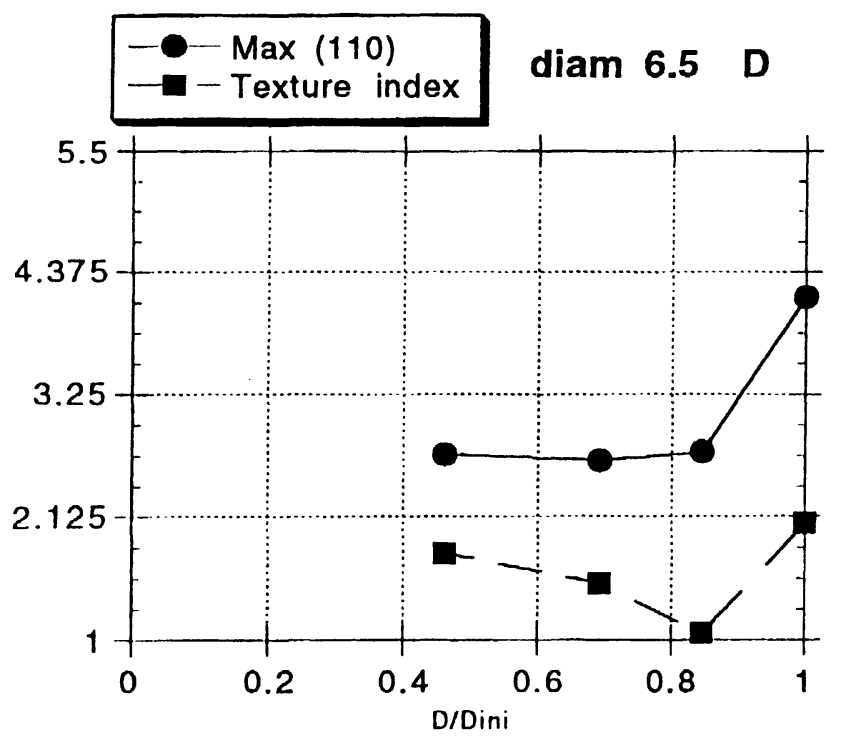

Figure 13 Texture gradient at the diameter $6.5 \mathrm{~mm}$ for D. 


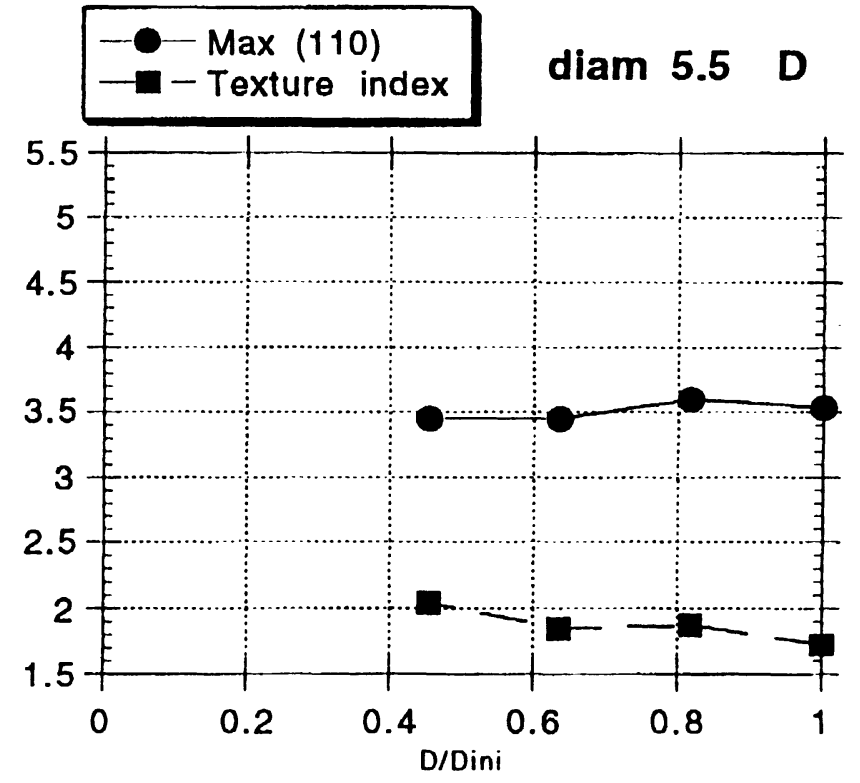

Figure 14 Texture gradient at the diameter $5.5 \mathrm{~mm}$ for D.

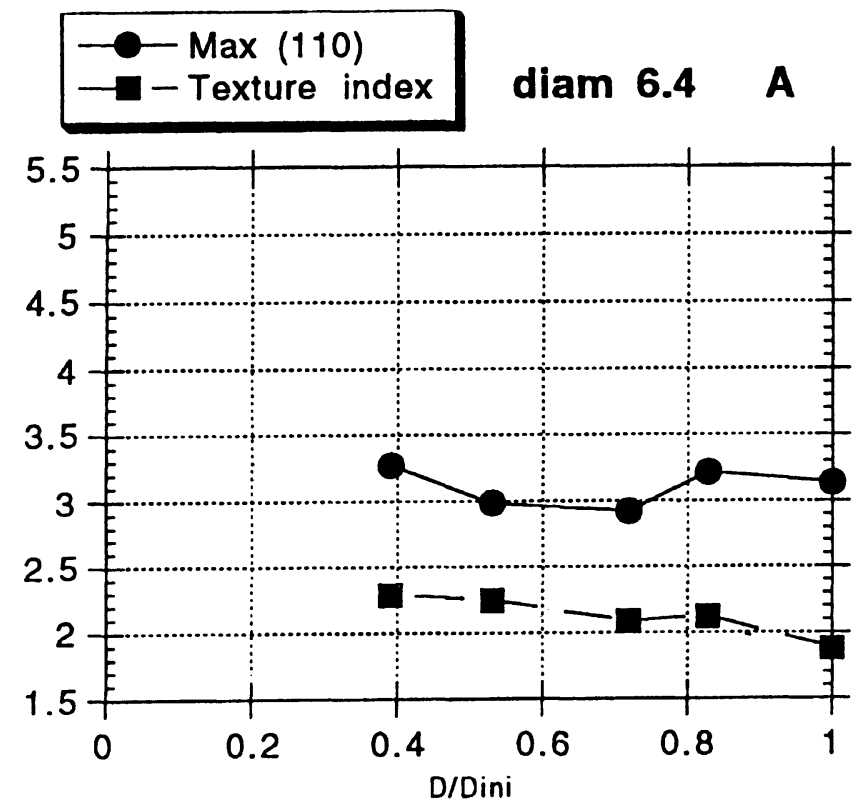

Figure 15 Texture gradient at the diameter $6.4 \mathrm{~mm}$ for A. 


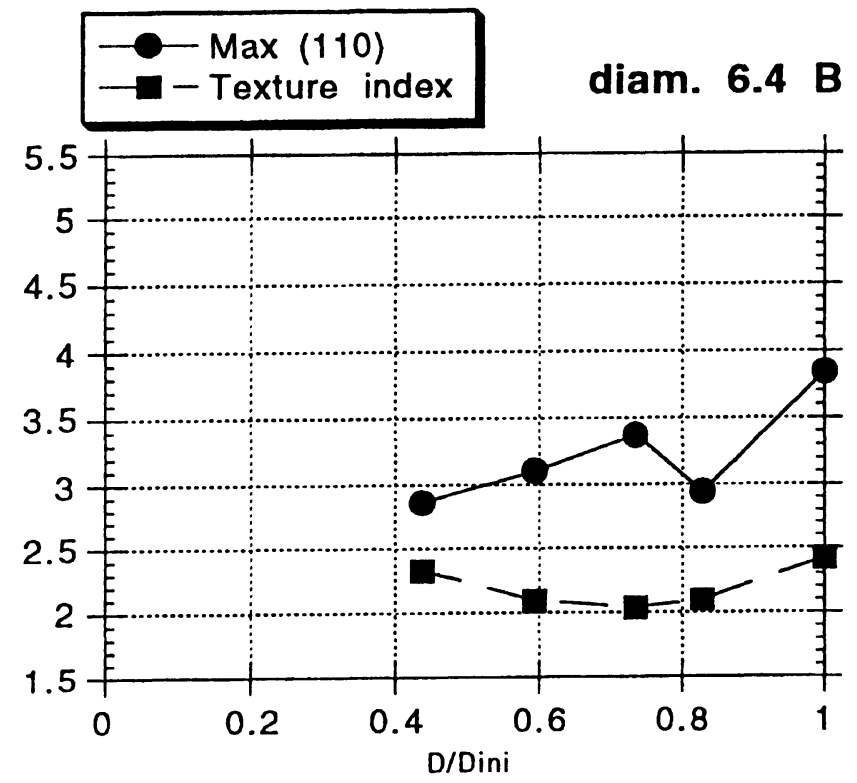

Figure 16 Texture gradient at the diameter $6.4 \mathrm{~mm}$ for $\mathrm{B}$.

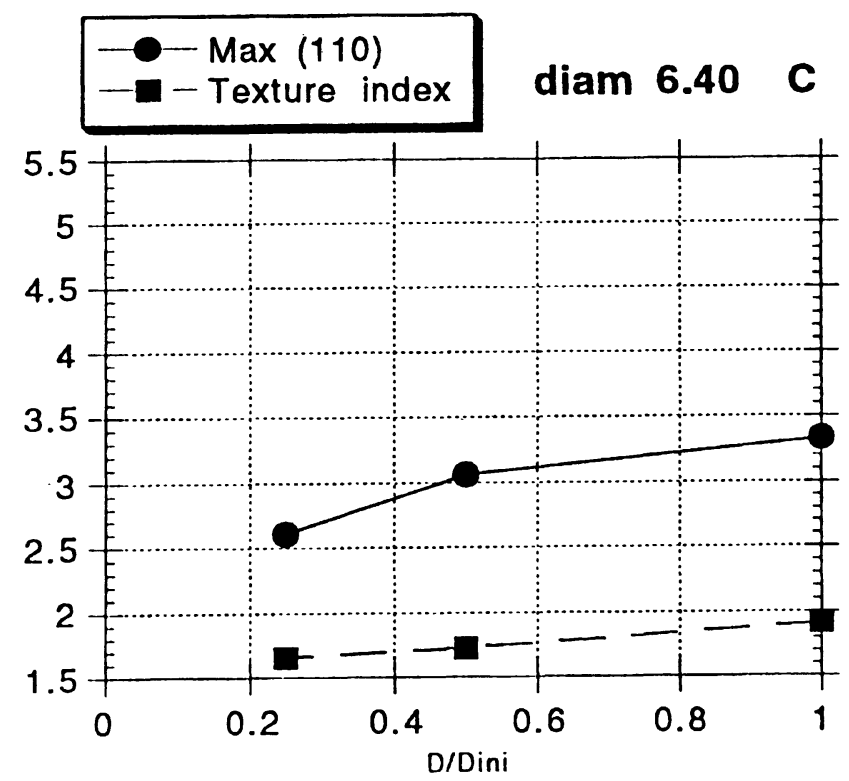

Figure 17 Texture gradient at the diameter $6.4 \mathrm{~mm}$ for C. 


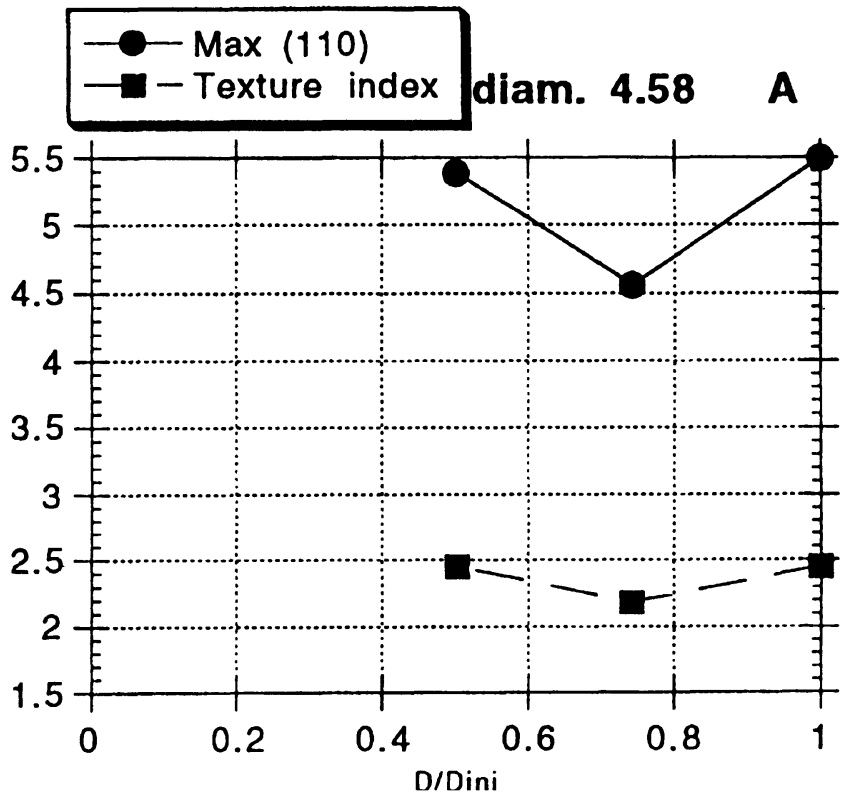

Figure 18 Texture gradient at the diameter $4.58 \mathrm{~mm}$ for A.

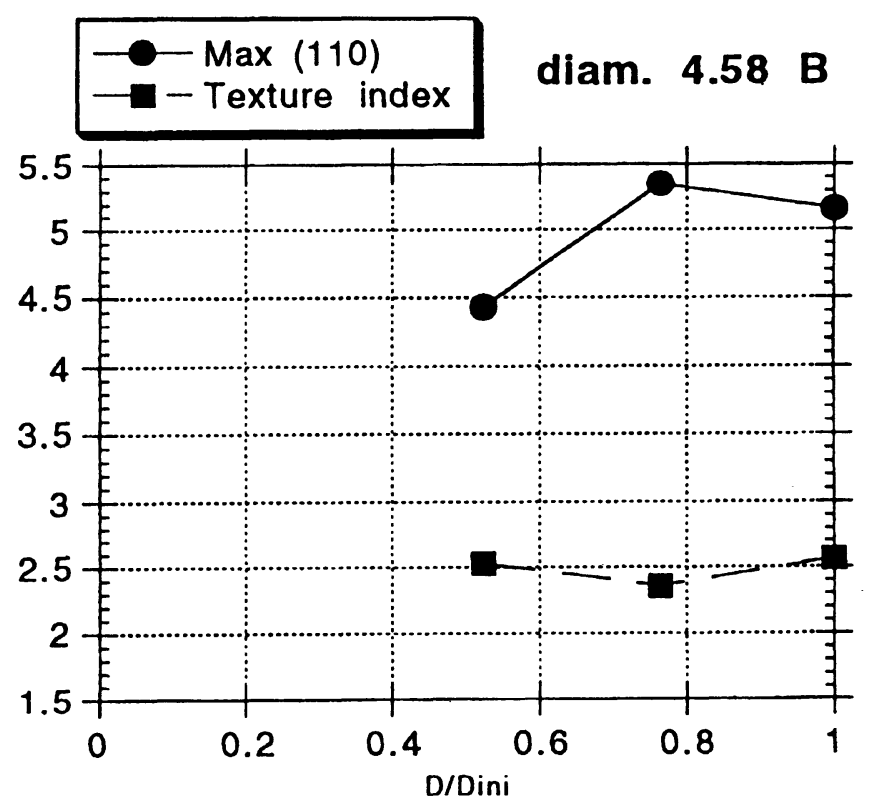

Figure 19 Texture gradient at the diameter $4.58 \mathrm{~mm}$ for B. 


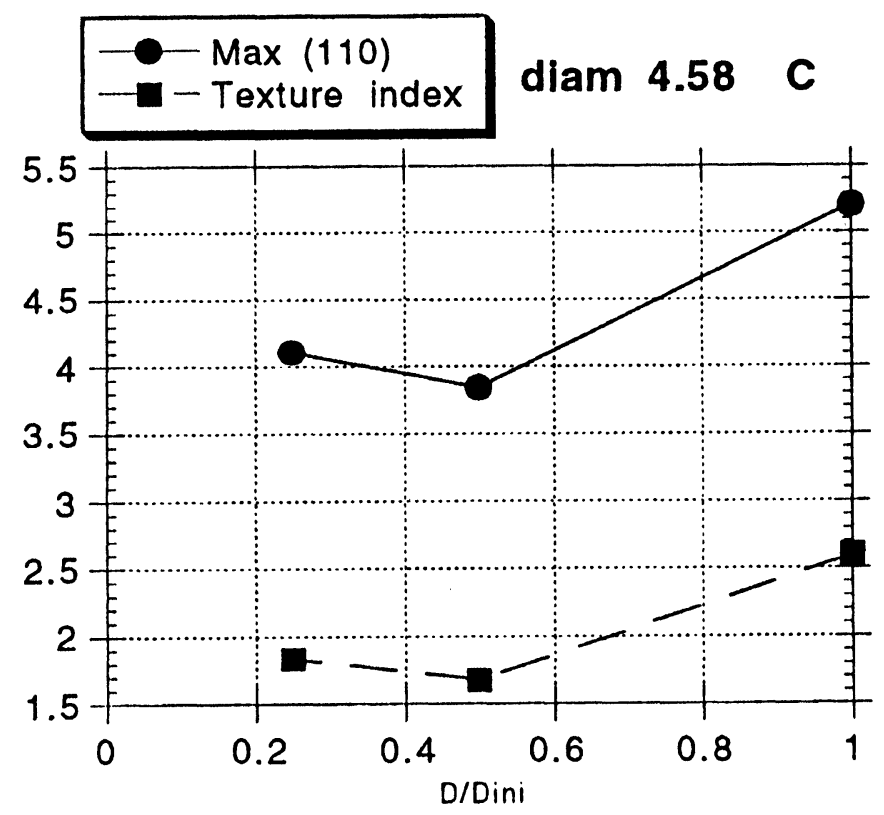

Figure 20 Texture gradient at the diameter $4.58 \mathrm{~mm}$ for $\mathrm{C}$.

Table 1 Summarizing table of the studied materials.

\begin{tabular}{cllcccc}
\hline Designation & Drawing & Speed & Grain size & Reduction rate & Reduction limit & Rem. \\
\hline A & alternate & industrial & $30 \mu$ & $20 \%$ & $99.44 \%$ & \\
B & alternate & industrial & $10 \mu$ & $20 \%$ & $99.65 \%$ & \\
C & unidirectionnal & industrial & $10 \mu$ & $20 \%$ & $99.12 \%$ & \\
D & unidirectionnal & slow & $10 \mu$ & $30 \%$ & & covered \\
E & unidirectionnal & industrial & & $20 \%$ & & with Cu \\
\hline
\end{tabular}

B. The total number of individual orientations differs slightly from one sample to the other because this number is set by the used computer code, according to some characteristics of the density function itself, like the minimum value per discrete orientation box in the Euler space.

As the Interstitial Free Steels have a bcc structure, there are with the $<111>$ densiest direction as glide direction 3 possible families of gliding systems according to the $\{110\}$, $\{112\},\{123\}$ planes.

The modellings were carried out using first the $\{110\}<111>$ system alone, then combined with the other possible gliding system, the CRSS being all set equal to 1 . The best results have been obtained by combining the 3 possible gliding systems and it turned out that it is always the planes with the highest indices which have the highest activity rate. Both observations tend to prove that the actual deformation mode is the pencil glide. Contrarily to Taylor simulations of cold rolling textures, the Taylor simulations of drawing textures yield textures which are smoother than the experimental ones, as can be seen e.g. on the normalised (110) PFs (Figures 21-24). Similar results 


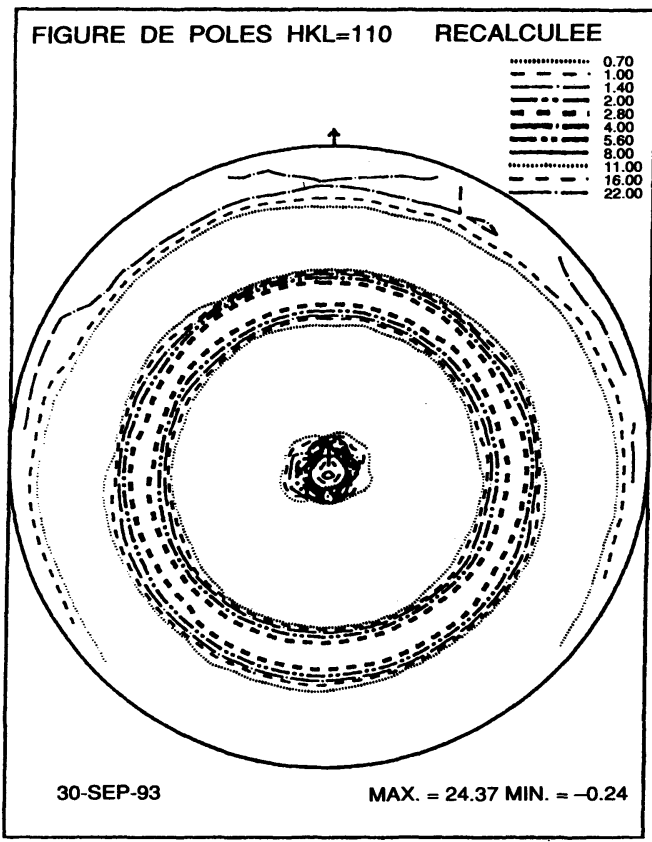

Figure 21 (110) recalculated PF for $B$ at the end of the deformation.

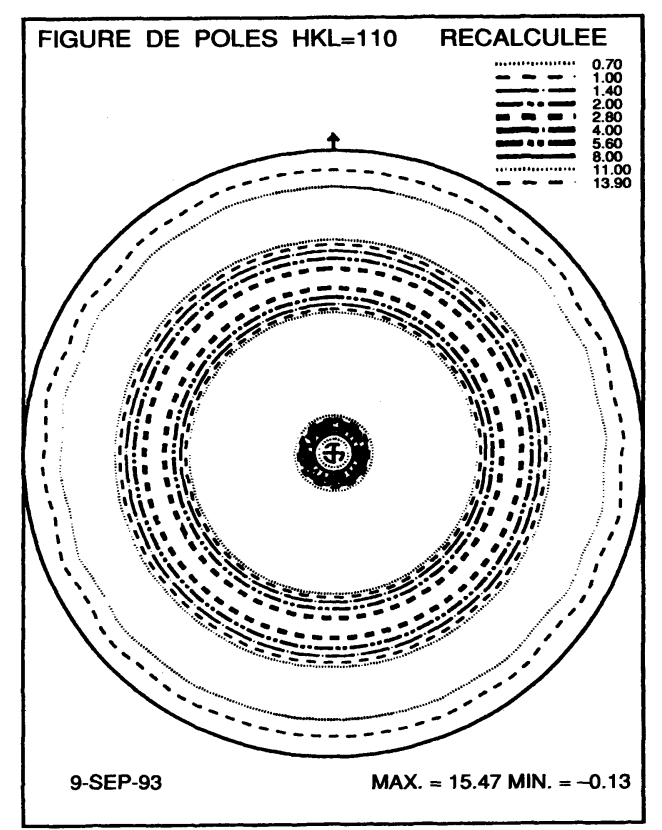

Figure 22 Taylor simulation for B at the same deformation degree than Figure 21. 


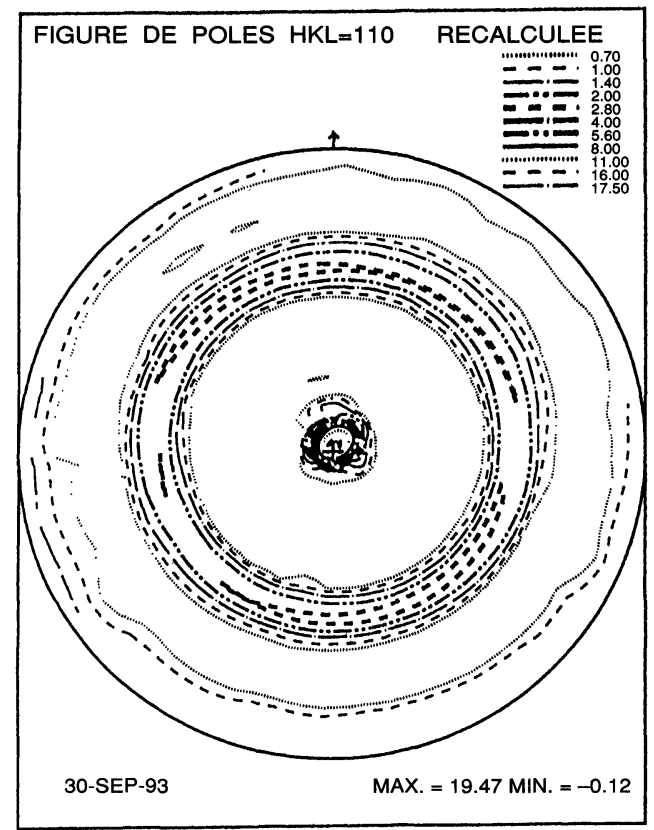

Figure 23 (110) recalculated $\mathrm{PF}$ for $\mathrm{A}$ at the end of the deformation.

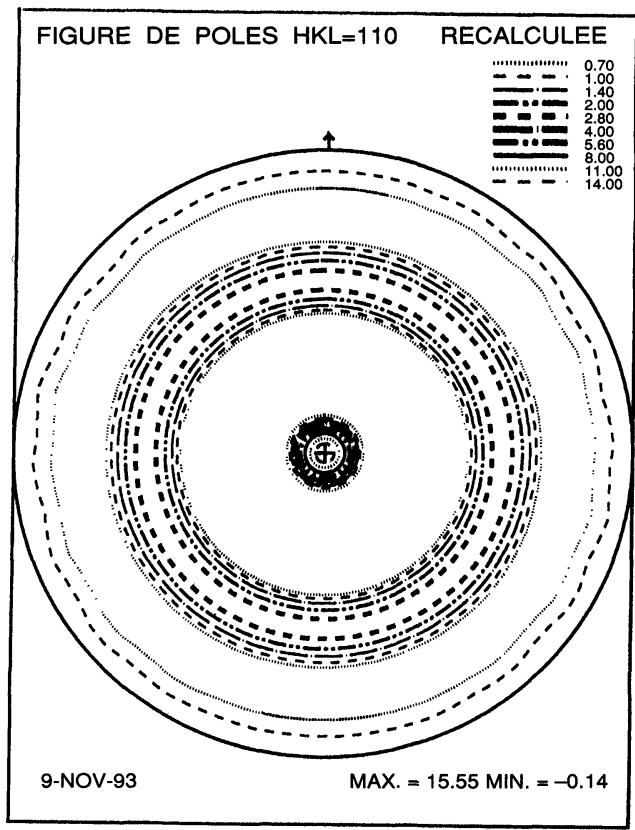

Figure 24 Taylor simulation for $\mathrm{A}$ at the same deformation degree than Figure 23. 
have been obtained by Arminjon et al. (1990) in the modelling of uniaxial tensile deformation of steels, for which the simulated textures were also smoother than the experimental ones.

\section{DISCUSSION}

When a metal with a random or smooth initial texture undergoes large deformations in a forming process, the final texture will present the symmetries of the forming process. So in the case of drawing the wire axis is a rotational symmetry axis of the texture (Coulomb (1972), Leber (1961)).

The textures observed in wires are fibre textures, characterized by a specific crystal direction $\langle\mathrm{hkl}>$ parallel to the wire axis. Drawn wires with a BCC structure show a $<110\rangle$ fibre, the rotational symmetry of the texture appearing clearly on the PFs (Figures 11-12).

Gliding is the only deformation mechanism that the material has to accommodate the plastic strain. Thus there won't be a large variety of possible wire drawing textures. All the authors quoted in the literature agree to say that there is a $<110\rangle$ fibre texture in drawing. Zenlyanskov et al. (1977) find a similar double axial texture $\langle 110\rangle+<114\rangle$ which is formed during drawing. Montesin et al. $(1990,1991)$ find a $\{100\}$ plane tangent to the wire surface during drawing.

In our case we obtained a $<110\rangle$ fibre texture. This fibre starts to build up in the very first percentages of deformation as a partial fibre texture and is complete after some drawing passes (50\% reduction). With increasing reduction, it keeps growing and sharpening up to the drawability limit of the material.

We did not find clear evidence of a texture gradient. The small texture variations along the radius of the wire could not affect significantly their mechanical properties which are influenced essentially by the surface state of the material. This surface state is governed by the die/metal friction which may be modified according to lubricant.

The results of texture modelling show that the Taylor model gives quite a good description of the texture evolution but it underestimates the texture sharpness.

\section{CONCLUSION}

The texture evolutions of low carbon steels during drawing are a function of the grain size of the starting material and of the deformation degree. This is due to the fact that gliding is the only deformation mechanism to accommodate the plastic strain. One of the main interest of the study of drawing texture for BCC materials lies in the possibility to include the texture function in the calculation of residual stresses in wires.

\section{References}

1. Arminjon, M. and Donadille, C. (1990). Mémoires et Etudes Scientifiques Revue de Métallurgie, Juin, 359-381.

2. Beautemps, J. and Jacquier, M. O. (1976). Métaux Corrosion-Industrie $\mathrm{N}^{\circ}$ 607, Mars.

3. Bunge, H. J. (1969). Mathematische Methoden der Texturanalyse, Akademie-Verlag, Berlin.

4. Coulomb, P. (1972). Les textures dans les métaux de réseau cubique Dunod Paris.

5. Embury, J. D. and Fischer, R. M. (1966). Acta. Met., 14, 147.

6. Guojn, H. and Hongru, Z. (1990). Wire Industry, Sept., 662-664. 
7. Langouche, F., Aernoudt, E. and Van Houtte, P. (1989). J. Appl. Cryst., 22, 533-538.

8. Lankford, G. (1970). Metallurgical Transactions, 1, Feb., 465-477.

9. Leber, S. (1961). Transactions of the ASM, 53, 687-713.

10. Montesin, T. and Heizmann, J. J. (1990). Revue de métallurgie Avril, 217-232.

11. Montesin, T., Heizmann, J. J. and Pelletier, J. B. (1991). Wire J. Int., 24-33.

12. Montesin, T., Heizmann, J. J., Addellaoui, A. and Pelletier, J. B. (1992). W. A. I. Joint Regional Meeting Rosemont.

13. Nakajima, S. and Okazaki, H. (1967). Trans of the Met. Soc. of AIME, 239, July, 1060-1065.

14. Rieck, G. D. and Koster, A. S. (1965). Trans of the Met. Soc. of AIME, 233, April, 770-772.

15. Zenlyanskov, V. A., Zlatoustovskiy, D. M., Nemkina, E. D. and Gubchevskiy, V. P. (1977). Fiz. Metal Metalloved 44, $\mathrm{n}^{\circ} 2,365-372$. 